\title{
Prognostic Significance of Hyponatremia Leukocytosis, Hypomagnesemia, and Fever after Aneurysmal Subarachnoid Hemorrhage
}

\author{
Vrsajkov Vladimir ${ }^{1}$ Jovanović Gordana ${ }^{2}$ Galešev Marija ${ }^{2}$ Uvelin Arsen ${ }^{1}$ Glišić Dunja ${ }^{1}$ \\ Pantić-Vrsajkov Jelena ${ }^{3}$
}

${ }^{1}$ Clinical Centre of Vojvodina, Emergency Centre, Novi Sad, Serbia

${ }^{2}$ Department of Anesthesia and Intensive Care, Clinical Centre of

Vojvodina, Novi Sad, Serbia

${ }^{3}$ Health Care Centre "Novi Sad," Novi Sad, Serbia

Address for correspondence Vrsajkov Vladimir, MD, PhD, Emergency Centre, Clinical Centre of Vojvodina, Hajduk Veljkova 1-7, 21000 Novi Sad, Serbia (e-mail: vladimir.vrsajkov@gmail.com).

Indian J Neurosurg 2015;4:69-73.

\begin{abstract}
Background Early identification of patients at an increased risk for delayed cerebral ischemia $(\mathrm{DCl})$ and poor outcome could allow more aggressive therapy and influence better outcome. The aim of this study was to determine a predictive association of hyponatremia, hypomagnesemia, fever, and leukocytosis with $\mathrm{DCl}$ and poor outcome. Patients and Methods We prospective enrolled 68 patients with subarachnoid hemorrhage (SAH) treated from March 2011 to May 2013. Serum levels of sodium, magnesium, and leukocyte count were determined at least once a day during the first 10 days after $\mathrm{SAH}$. All patients underwent noncontrast computed tomography (CT) scan $9 \pm 2$ days after SAH. DCl was defined as one or more of the next parameters: a new focal neurological deficit, decline for two or more points on the modified Glasgow Coma Scale or a new hypodensity on CT scan. The outcome was assessed after 6 months using the extended Glasgow Outcome scale.

Results Overall, $48 \%$ of the patients recruited had DCI. Ramachandraiah logistic

Keywords

- subarachnoid hemorrhage

- cerebral ischemia

- Glasgow outcome scale

- Hyponatremia

- magnesium regression model showed significant impact of hyponatremia $(p=0.036$; odds ratio $[\mathrm{OR}]=4.08$; $95 \%$ confidence interval $[\mathrm{Cl}]=1.09-15.26)$ on $\mathrm{DCl}$ and poor outcome $(p=0.034 ; \mathrm{OR}=5.11 ; 95 \% \mathrm{Cl}=1.13-23.14)$. We obtained strong correlation of leukocytosis $(p=0.013)$ with $\mathrm{DCl}$ and poor outcome $(p=0.016)$. Association of noninfectious fever and hypomagnesemia with $\mathrm{DCl}$ existed, but it was not significant enough.

Conclusion Our results confirmed the association of hyponatremia and leukocytosis with greater risk of developing $\mathrm{DCl}$ and poor clinical outcome.
\end{abstract}

\section{Introduction}

Delayed cerebral ischemia (DCI) is a major factor in morbidity and mortality after aneurysmal subarachnoid hemorrhage (SAH). Numerous studies have reported various factors for identifying patients at risk of developing DCI. General consensus was not established and further studies are needed. ${ }^{1,2}$

received

October 22, 2014

accepted

May 25, 2015

published online

July 28, 2015
DOI http://dx.doi.org/

10.1055/s-0035-1558961. ISSN 2277-954X.
Hyponatremia occurs in 30 to $50 \%$ of patients with $\mathrm{SAH}^{3-5}$ The etiology of hyponatremia is still unclear, but it is probably mediated by hypothalamic injury secondary to SAH.

Regardless of the cause of hyponatremia, it can worsen the patient's neurological condition after SAH and has negative effect on morbidity and mortality. ${ }^{5,6}$ First studies showed significant relationship between hyponatremia and

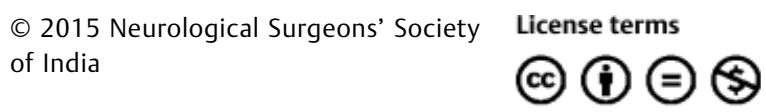


the occurrence of cerebral ischemia but later studies failed. ${ }^{3,7,8}$ However, in the recent years, some new results (Chandy et al and Zheng et al) showed significant value of hyponatremia in predicting neurological complications and poor outcome. ${ }^{4,9,10}$

Hypomagnesemia occurs in $38 \%$ of patients with SAH. Data by van den Bergh et al have associated it with the development of DCI and poor outcome. ${ }^{11}$ These results inspired many studies addressing therapeutic administration of intravenous magnesium, but the possible predictive role of hypomagnesemia has not been investigated enough. Few studies investigated this and results were inconsistent. $^{10,12,13}$

Recent data confirmed correlation between increased levels of inflammatory markers and the development of neurological complications. ${ }^{14} \mathrm{Few}$ studies showed positive predictive value of leukocytosis but influence of early (first 3 days after $\mathrm{SAH}$ ) leukocytosis and cat off value of leukocyte count are still matter of debate. ${ }^{14-17}$

Some investigations reported possible predictive value of fever in patients with SAH. ${ }^{18,19}$ However, significant issues remain unresolved in previous studies. Among them are the differences in the predictive value of noninfectious and infectious fever and the upper temperature limit that would have the greatest statistical significance.

The aim of our study was to examine the clinical influence of different parameters of metabolic disbalance on emergence of $\mathrm{DCI}$ and poor outcome.

\section{Patients and Methods}

This prospective, observational study was conducted in Clinical center of Vojvodina between March 2011 and May 2013. Overall 68 patients were enrolled in the study. The approval of the Ethics Committee of Clinical center of Vojvodina was obtained. Inclusion criteria were as follows: (1) Admission to Clinical center of Vojvodina within the first 72 hours after aneurysmal rupture. (2) A World Federation of Neurosurgical Societies (WFNS) score of I-IV

Exclusion criteria were as follows: (1) cerebral aneurysm of traumatic, fusiform or mycotic origin; (2) WFNS score of $\mathrm{V}$; (3) concomitant severe neurological, psychiatric, or medical illnesses; and (4) angiographic vasospasm on admission computed tomographic (CT) scan.

Any history of arterial hypertension and smoking habits were recorded on admission. Arterial hypertension was defined as the use of antihypertensive therapy last 3 months before SAH.

Level of sodium, magnesium, and white blood cells count in the blood was assessed every 12 hours. Duration of hyponatremia, leukocytosis, and hypomagnesemia considered clinically significant was at least 24 hours. Hyponatremia was defined as serum sodium level $<135 \mathrm{mmol} / \mathrm{L}$, hypomagnesemia as serum magnesium level $<0.70 \mathrm{mmol} / \mathrm{L}$ and leukocytosis as serum leukocyte count $>12 \times 10^{9} / \mathrm{L}$ occurring at any point in time during the first 10 days after $\mathrm{SAH}$. Patients were classified as febrile if body temperature $\geq$ $37.8^{\circ} \mathrm{C}$ was documented on at least two daily measurements.
All patients underwent noncontrast CT scan $9 \pm 2$ days after SAH. DCI was defined as one or more of a new focal neurological deficit, decline for two or more points on the mGCS scale or new hypodensities on CT scan not visible on admission or postoperative scan, not associated with initial hemorrhage, an intracerebral hematoma, placement of a ventricular catheter, metabolic disturbance, infection, or hypoxia. Cerebral ischemia was verified with CT scan by a study independent neuroradiologist. The outcome was assessed at 6 months after aneurysmal rupture with an extended Glasgow Outcome scale (eGOS). ${ }^{20}$ Poor outcome was defined as 1-4 points on eGOS scale.

\section{Statistical Analysis}

For statistical analysis SPSS 20.0 was used. Differences between the groups were analyzed by Student $t$-test for normal distribution data and the Mann-Whitney $U$-test for data without normal distribution. For nonparametric data, the chi-square test was used. Logistic regression model was used to evaluate the impact of nondependent variables on $\mathrm{DCI}$ and poor outcome.

\section{Results}

The study included 68 patients with aneurysmal SAH. Positive finding of DCI was found in 33 patients (48\%).

- Table 1 shows the influence of demographic characteristics, neurological status, smoking, and hypertension on development of DCI. In the group of patients with $\mathrm{DCI}$, there was significantly higher WFNS score than in the group without delayed ischemia. Arterial hypertension in medical history has been shown to have significant impact on the development of DCI. Statistically significant differences were obtained for length of stay in the intensive care unit $(p=0.001)$ and on hospital ward $(p=0.025)$ between patients with and without DCI.

Nine patients were excluded because of the fever of infectious origin (positive microbiological testing).

- Table 2 shows the influence of fever, leukocytosis, hyponatremia, and hypomagnesemia on the development of DCI.

Statistically significant difference between DCI and DCI absent groups was obtained for hyponatremia and leukocytosis.

Logistic regression model showed significant impact of hyponatremia ( $p=0.036$; $\mathrm{OR}=4.08 ; 95 \% \mathrm{CI}=1.09-15.26$ ) on the development of DCI.

Six months outcome on eGOS scale was $4.27 \pm 2.42$ in the group with $\mathrm{DCI}$ and $5.83 \pm 2.32$ in the group without DCI $(p=0.004)$.

Overall, 15 patients died, while two had severe neurological damage. A good outcome (5-8 points on eGOS scale) was achieved in 51 patients.

- Table 3 shows the influence of demographic characteristics, neurological status, smoking, and arterial hypertension in the development of poor outcome.

In the group of patients with poor outcome, WFNS score was significantly higher than in the group with good 
Table 1 Demographic characteristics, neurological status, smoking, and hypertension

\begin{tabular}{|l|l|l|l|}
\hline Variables & DCl & DCl absent & $p$-Value \\
\hline Gender, male/female $(n)$ & $11 / 22$ & $15 / 20$ & 0.41 \\
\hline Age & $56.34 \pm 9.6$ & $53.45 \pm 10.0$ & 0.32 \\
\hline Location of the aneurysm, a/p $(n)$ & $29 / 4$ & $29 / 6$ & 0.55 \\
\hline WFNS score on admission $(n)$ & $2.42 \pm 1.25$ & $1.83 \pm 1.07$ & 0.038 \\
\hline Intraventricular hemorrhage, yes/no $(n)$ & $18 / 15$ & $16 / 19$ & 0.46 \\
\hline Smoking, yes/no & $23 / 10$ & $24 / 11$ & 0.92 \\
\hline HTA, yes/no & $24 / 9$ & $15 / 20$ & 0.013 \\
\hline
\end{tabular}

Abbreviations: DCl, delayed cerebral ischemia; SD, standard deviation; WFNS, World Federation of Neurosurgical Societies.

Note: Data are expressed as numbers or mean \pm SD.

Table 2 Influence of fever, leukocytosis, hyponatremia, and hypomagnesemia

\begin{tabular}{|l|l|l|l|}
\hline Variables & DCI & $\begin{array}{l}\text { DCI } \\
\text { absent }\end{array}$ & $\begin{array}{l}p \\
\text { Value }\end{array}$ \\
\hline Noninfectious fever, yes/no $(n)$ & $20 / 8$ & $17 / 14$ & 0.19 \\
\hline Hyponatremia, yes/no $(n)$ & $19 / 14$ & $7 / 28$ & 0.01 \\
\hline Leukocytosis, yes/no $(n)$ & $24 / 9$ & $15 / 20$ & 0.013 \\
\hline Early leukocytosis, yes/no $(n)$ & $18 / 15$ & $11 / 24$ & 0.054 \\
\hline Hypomagnesemia, yes/no $(n)$ & $20 / 13$ & $16 / 19$ & 0.26 \\
\hline
\end{tabular}

Abbreviation: $\mathrm{DCl}$, delayed cerebral ischemia.

Note: Data are expressed as numbers.

outcome. Arterial hypertension also had a significant impact on the development of poor outcome.

- Table 4 shows the influence of fever, leukocytosis, hyponatremia, and hypomagnesemia on poor outcome. Statistically significant differences were obtained for hyponatremia and leukocytosis.

Logistic regression model has shown significant impact of hyponatremia $(p=0.034 ; \mathrm{OR}=5.11 ; 95 \% \mathrm{CI}=1.13-23.14$ ) on poor outcome.

\section{Discussion}

This study undertaken over 2 years and included 68 patients with SAH.
Hyponatremia after $\mathrm{SAH}$ is present in 28 to $55 \%$ of patients. We got similar results (38\%). These results significantly overlap our retrospective study (2010) with $39 \%$ of patients with hyponatremia. ${ }^{3,5,21}$ Laboratory control of the serum and urine osmolarity, sodium in urine and central venous pressure were standard measures in prevention of fluid restriction, maintenance of euvolemia, and therapy guide for daily fluid intake in the case of hyponatremia. Hypertonic saline solution (3\%) was used to correct hyponatremia below $130 \mathrm{mmol} / \mathrm{L}$ or if it continued for more than 36 hours.

Focus of the study was a possible role of hyponatremia in prediction of cerebral ischemia and poor outcome. Our research from 2011 showed a positive correlation between hyponatremia and vasospasm, but in the context of clinical symptoms of $\mathrm{DCl}^{5}$ By comparing time of occurrence of hyponatremia and DCI (decrease of serum sodium level between 7 th and 10th day, when we had the most of neurological complications) we did not get statistically significant correlation, which contradicts the results of Ramachandraiah and Chandy. ${ }^{22}$ The cause of these differences could possibly be explained by designs of the following studies: they used sodium level below 135 or $4 \mathrm{mmol} / \mathrm{L}$ decrease from admission level as referent values. A significant difference of the outcome according to eGOS scale confirms the results of study of Saleh et al and our results from 2011.,10

We obtained $53 \%$ of patients with hypomagnesemia, which is much more than van den Bergh (38\%),

Table 3 Influence of demographic characteristics, neurological status, smoking, and arterial hypertension

\begin{tabular}{|l|l|l|l|}
\hline Variables & Poor outcome & Good outcome & $p$ Value \\
\hline Gender male/female $(n)$ & $8 / 9$ & $18 / 33$ & 0.38 \\
\hline Age & $58.29 \pm 9.62$ & $53.82 \pm 9.79$ & 0.10 \\
\hline WFNS score on admission & $2.65 \pm 1.27$ & $1.94 \pm 1.12$ & 0.03 \\
\hline Intraventricular hemorrhage, yes/no $(n)$ & $10 / 7$ & $24 / 27$ & 0.42 \\
\hline Smoking, yes/no $(n)$ & $13 / 4$ & $34 / 17$ & 0.44 \\
\hline HTA, yes/no $(n)$ & $14 / 3$ & $25 / 26$ & 0.01 \\
\hline
\end{tabular}

Abbreviations: DCl, delayed cerebral ischemia; SD, standard deviation; WFNS, World Federation of Neurosurgical Societies.

Note: Data are expressed as numbers or mean \pm SD. 
Table 4 Influence of fever, leukocytosis, hyponatremia, and hypomagnesemia on poor outcome

\begin{tabular}{|l|l|l|l|}
\hline Variables & Poor outcome & Good outcome & $p$-Value \\
\hline Noninfectious fever, yes/no $(n)$ & $11 / 4$ & $26 / 18$ & 0.32 \\
\hline Hyponatremia. yes/no $(n)$ & $12 / 5$ & $14 / 37$ & 0.002 \\
\hline Leukocytosis, yes/no $(n)$ & $14 / 3$ & $25 / 26$ & 0.016 \\
\hline Early leukocytosis, yes/no $(n)$ & $10 / 7$ & $19 / 32$ & 0.11 \\
\hline Hypomagnesemia, yes/no (n) & $11 / 6$ & $25 / 26$ & 0.26 \\
\hline
\end{tabular}

Note: Data are expressed as numbers.

Collignon et al (32\%), Mahdi et al (22\%). ${ }^{11-13}$ In this study, hypomagnesemia in the first 72 hours after rupture or between 4th and 10th day after rupture did not show significant correlation with DCI and poor outcome. Our results are similar to the results of Collignon et al, but they differ from the results of van den Bergh et al, Mahdi et al, Saleh et al. ${ }^{10-13}$ Final conclusion about the predictive value of hypomagnesemia cannot be made because of mutually exclusive results of studies and because none of these studies, including ours, do not have samples bigger than 128 patients. A large number of patients with hypomagnesemia indicate the importance of regular monitoring of the magnesium blood level after cerebral aneurysm rupture, and necessity of correcting pathological values.

Study of Dhar and Diringer (2008) showed correlation between some systemic inflammatory response syndrome parameters (SIRS) and DCI. ${ }^{23}$ Our goal was to investigate possible role of leukocytosis in appearance of DCI and poor outcome. Studies of McGirt et al (2003), Choi et al (2007), and Van Assche et al (2006) confirmed connection between leukocytosis and symptomatic vasospasm, but potential impact on the final outcome is not sufficiently explored. ${ }^{15-17}$ These studies used level of $15 \times 10^{9}$ as reference, but we used $12 \times 10^{9}$ because that value is the diagnostic limit for SIRS. Because of that, we had $57 \%$ of patients with leukocytosis in our study, and we have shown a statistically significant correlation between leukocytosis and cerebral ischemia and negative outcome. Comparing leukocytosis in the first 3 days after rupture, we got difference between the groups but not statistically significant. Our results show the potential benefit of $12 \times 10^{9}$ limit, but new researches with greater number of participants are needed to give the right answer. Although multivariate analysis did not show predictive significance of leukocytosis our results confirmed potential of it, and 41 to $72 \%$ of patients with SAH had increased body temperature. Studies of Oliveira-Filho et al (2001), Naidech et al (2007), and Douds et al (2012) show a significant correlation between fever and symptomatic vasospasm and poor outcome. ${ }^{18,19,24}$ These studies used body temperature between $37.5^{\circ} \mathrm{C}$ and $38.3^{\circ} \mathrm{C}$ as a limit of fever. None of the studies investigated this subject made a difference between fever of infectious and noninfectious origin. ${ }^{18,19,24}$ Our goal was to determine the relationship between fever of noninfectious genesis between day 4 and day 9 following the rupture and DCI or poor outcome. This fever is an important part of systemic inflammatory response in this period in patients with signs of DCI. We obtained $54 \%$ of patients with noninfectious fever, although other studies had. 33 to $55 \%{ }^{18,19}$ More patients with fever in our study can be explained by different study design and different fever limit. Our results do not show significant correlation between fever of noninfectious genesis and DCI and poor outcome. Studies of Douds et al and Oliveira-Filho et al had no proper distinction between infectious and noninfectious fever, and Naidech et al had different methodology ${ }^{18,19,24}$ that would explain the differences with our results.

We are aware that this study has several limitations. The real problem is the relatively small number of patients. This is a consequence of incidence of SAH in our hospital and exclusion of patients of level V of WFNS scale from the study. Relatively small number of patients did not let us to define existence of time connection (when precisely hyponatremia or leukocytosis precede DCI).

\section{Conclusion}

These results are still very interesting, implying a clear benefit of hyponatremia above and leukocytosis as additional predictors of developing DCI. We consider that daily monitoring of serum sodium and leukocytes level in the early course of SAH treatment can contribute to identifying patients with high risk of neurological complications and poor outcome and bring potential benefit in treatment protocols.

\section{Note}

This article contains original unpublished work, not being submitted for publication elsewhere. Submission of the article has been approved by the authorities from University hospital and Clinical center Novi Sad, Serbia. The authors declare that there is no conflict of interests regarding the publication of this article. This is a joint statement of all the listed authors.

\section{References}

1 Harrod CG, Bendok BR, Batjer HH. Prediction of cerebral vasospasm in patients presenting with aneurysmal subarachnoid hemorrhage: a review. Neurosurgery 2005;56(4): 633-654, discussion 633-654 
2 Lazaridis C, Naval N. Risk factors and medical management of vasospasm after subarachnoid hemorrhage. Neurosurg Clin N Am 2010;21(2):353-364

3 Qureshi AI, Suri MF, Sung GY, et al. Prognostic significance of hypernatremia and hyponatremia among patients with aneurysmal subarachnoid hemorrhage. Neurosurgery 2002; 50(4):749-755, discussion 755-756

4 Chandy D, Sy R, Aronow WS, Lee WN, Maguire G, Murali R. Hyponatremia and cerebrovascular spasm in aneurysmal subarachnoid hemorrhage. Neurol India 2006;54(3):273-275

5 Vrsajkov V, Javanović G, Stanisavljević S, Uvelin A, Vrsajkov JP. Clinical and predictive significance of hyponatremia after aneurysmal subarachnoid hemorrhage. Balkan Med J 2012; 29(3):243-246

6 Levine JM. Critical care management of subarachnoid hemorrhage. Curr Treat Options Neurol 2009;11(2):126-136

7 Hasan D, Wijdicks EF, Vermeulen M. Hyponatremia is associated with cerebral ischemia in patients with aneurysmal subarachnoid hemorrhage. Ann Neurol 1990; 27(1):106-108

8 Wartenberg KE, Schmidt JM, Claassen J, et al. Impact of medical complications on outcome after subarachnoid hemorrhage. Crit Care Med 2006;34(3):617-623, quiz 624

9 Zheng B, Qiu Y, Jin H, et al. A predictive value of hyponatremia for poor outcome and cerebral infarction in high-grade aneurysmal subarachnoid haemorrhage patients. J Neurol Neurosurg Psychiatry 2011;82(2):213-217

10 Saleh A, Megahed M, Ibrahim K. Hypomagnesemia, Hyponatremia And Hypercholesterolemia On Admission As Prognostic Predictors For Patients With Subarachinoid Haemorrhage. The Internet Journal of Interventional Medicine. 2010;1(1):59-69

11 van den Bergh WM, Algra A, van der Sprenkel JW, Tulleken CA, Rinkel GJ. Hypomagnesemia after aneurysmal subarachnoid hemorrhage. Neurosurgery 2003;52(2):276-281, discussion 281-282

12 Collignon FP, Friedman JA, Piepgras DG, et al. Serum magnesium levels as related to symptomatic vasospasm and outcome following aneurysmal subarachnoid hemorrhage. Neurocrit Care 2004;1(4):441-448

13 Mahdi Z, Saberi H, Tabatabaei FSA, Tayebi MA, Habibi Z. Serum magnesium levels and clinical outcome of aneurysmal subarachnoid hemorrhage: a study in 60 patients. Tehran Univ Med J 2008;66(1):7-11
14 McMahon CJ, Hopkins S, Vail A, et al. Inflammation as a predictor for delayed cerebral ischemia after aneurysmal subarachnoid haemorrhage. J Neurointerv Surg 2013;5(6):512-517

15 McGirt MJ, Mavropoulos JC, McGirt LY, et al. Leukocytosis as an independent risk factor for cerebral vasospasm following aneurysmal subarachnoid hemorrhage. J Neurosurg 2003;98(6): $1222-1226$

16 Choi JH, Moon JG, Lee HK, Hwang DY, Kim CH, Choi JH. Correlation between Symptomatic Vasospasm and Leukocyte, Platelet Count Following Aneurysmal Subarachnoid Hemorrhage: Clinical Research. Korean J Cerebrovasc Surg. 2007;9(2): 135-142

17 Van Assche E, De Deyne C, Schneider I, Vander Laenen M, Heylen R. Early leukocytosis occurring after subarachnoid hemorrhage could be used as a predictor of later cerebral vasospasm: A-349. European J Anaesthesilogy 2006;23:9216

18 Oliveira-Filho J, Ezzeddine MA, Segal AZ, et al. Fever in subarachnoid hemorrhage: relationship to vasospasm and outcome. Neurology 2001;56(10):1299-1304

19 Douds GL, Tadzong B, Agarwal AD, Krishnamurthy S, Lehman EB, Cockroft KM. Influence of Fever and hospital-acquired infection on the incidence of delayed neurological deficit and poor outcome after aneurysmal subarachnoid hemorrhage. Neurol Res Int 2012;2012:479865

20 Jennett B, Snoek J, Bond MR, Brooks N. Disability after severe head injury: observations on the use of the Glasgow Outcome Scale. J Neurol Neurosurg Psychiatry 1981;44(4):285-293

21 Connolly ES Jr, Rabinstein AA, Carhuapoma JR, et al; American Heart Association Stroke Council; Council on Cardiovascular Radiology and Intervention; Council on Cardiovascular Nursing; Council on Cardiovascular Surgery and Anesthesia; Council on Clinical Cardiology.Guidelines for the management of aneurysmal subarachnoid hemorrhage: a guideline for healthcare professionals from the American Heart Association/ american Stroke Association. Stroke 2012;43(6):1711-1737

22 Ramachandraiah V, Chandy D. Hyponatremia and Cerebrovascular Spasm in Aneurysmal Subarachnoid Hemorrhage. Chest 2012; 142(4):371A

23 Dhar R, Diringer MN. The burden of the systemic inflammatory response predicts vasospasm and outcome after subarachnoid hemorrhage. Neurocrit Care 2008;8(3):404-412

24 Naidech AM, Bendok BR, Bernstein RA, et al. Fever burden and functional recovery after subarachnoid hemorrhage. Neurosurgery 2008;63(2):212-217, discussion 217-218 